\begin{tabular}{lccr} 
A R C H I V E & O F & M E C H A N I C A L & E N G I N E E R I N G \\
\hline VOL. LXI & 2014 & Number 1
\end{tabular}

10.2478/meceng-2014-0001

Key words: turbogenerator coil retaining rings, low cycle fatigue, machine safety, probability of failure, AMV method

\title{
RELIABILITY ASSESSMENT OF A TURBOGENERATOR COIL RETAINING RING BASED ON LOW CYCLE FATIGUE DATA
}

\begin{abstract}
Turbogenerator coil retaining rings are shrunk-fitted onto the rotor over the coils, in order to restrain them against the centrifugal force. They are typically subjected to low cycle fatigue, with a cycle being completed at every machine switch-on and switch-off. The subject of this paper consists in the determination of the failure probability of a coil retaining ring. The failure mode of the ring cracking, when it swells in tension, due to the centrifugal force is here considered. The reliability assessment is preceded by the study of the input variables affecting the low-cycle fatigue load and of their stochastic distributions. This question is tackled by the experimental determination of the static, cyclic and fatigue curves of the involved material and by the application of a statistical model to compute related parameters and their standard deviations. Upon the determination of variable distributions, the probability of failure is estimated in the form of a cumulative distribution function by a computationally efficient methodology, based on the Advanced Mean Value approach. The obtained results account for the material response and the local stressstrain states at the most loaded coil retaining ring region. The determined probability at the end of the machine life, in the order of $10^{-12}$, is compatible with reference values for structures under fatigue in the mechanical and aeronautical fields.
\end{abstract}

\section{Introduction}

The main components of a turbogenerator, the rotor and the coil retaining ring (CRR), are typical examples of devices experiencing low cycle fatigue (LCF). They are incredibly large sized machines: the rotor has $1.2 \mathrm{~m}$ diameter and $4.5 \mathrm{~m}$ length, while the CRRs applied at the rotor ends, have a quite short thickness, about $80 \mathrm{~mm}$. The LCF load acting on the CRR arises from the constructive details of a turbogenerator, which can be briefly summarized as follows. The rotor exhibits uniformly spaced longitudinal slots. Copper

* Department of Industrial Engineering (DIN), University of Bologna, Bologna, Italy; E-mail: giorgio.olmi@unibo.it 
conductors and insulating materials are packed into the slots and emerge at their ends, to form a coil. As the rotor spins (nominal speed of 3,000 rpm), the copper conductors are subjected to high centrifugal forces and must be restrained. Along most of the rotor length, they are constrained by metal wedges applied along the slots. At the ends, constraining is achieved by the shrink fit of CRRs onto the rotor body over the coils. Each CRR is finally fixed by locking keys against displacements in the axial direction. Fig. 1 (a) shows the typical layout of a turbogenerator, with data on its main dimensions. Details regarding the copper windings at rotor extremity before CRR shrink-fitting, and coil constraining by metal wedges are shown in Fig. 1 (b). The shapes of locking keys and related grooves are finally highlighted in Fig. 1 (c). As the rotor spins, a strong centrifugal force is transmitted to the CRR by the copper masses. As a consequence, the ring swells in tension, and the stress state at its central part (at the inner side) reaches its maximum values. Subsequently, when the machine is stopped, the centrifugal force decreases to zero and the stress is released. Consequently, a LCF cycle is completed at every machine switch on and switch off. A similar effect causes LCF on the rotor too, in particular, in the zone involved by the shrink-fit coupling. The expected number of cycles in the whole machine life is between 10,000 and 15,000. The consequences of an on service failure involving the rotor or the CRR may be very serious, since an explosion is likely to occur [1-2].

The performance and the resistance of an engineered system is often affected by several uncertainties [3]. Being able to manage and estimate the effects of uncertainty on the design performance is now becoming more and more important, since a not full comprehension may lead to unexpected failures. Probabilistic analyses are often based on the experimental knowledge of the structural behaviour [3]. In the turbogenerator field a reliability quantitative analysis is also important: the conventional approach to design requires the execution of deterministic calculations, based on the typical models for LCF, with the simulation of the stress-strain hysteresis loops at most stressed locations and the estimation of the residual life by the adopted strain-life model. Managing the uncertainties affecting this result and their effects means being able to determine the probability of failure for the most critical failure modes.

The aforementioned analysis can be implemented as follows. Let $\underline{U}$ be the vector of the $r$ input variables $U_{i}$ and let $Y$ be the output variable. Let $g$ be the functional relationship between the effect $Y$ and its causes $\underline{U}: Y=g(\underline{U})$. The goal of the probabilistic analysis consists in the determination of the probability $P$ of $Y \leq y$, given the distributions of the random inputs and being $y$ a threshold value. The curve relating $P$ to $y$, for different threshold values 

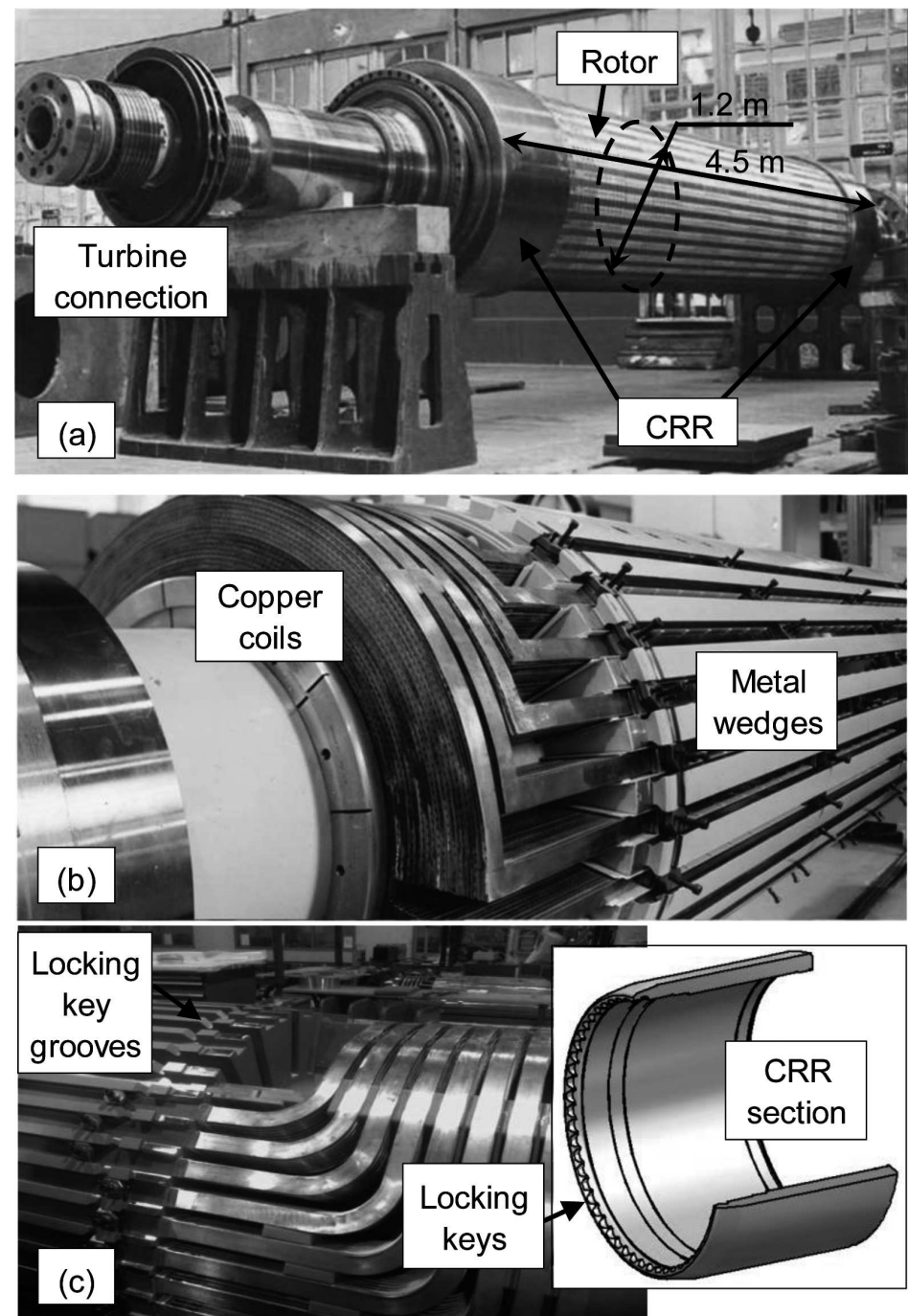

Fig. 1. Layout of a turbogenerator (a) and details on constraining strategies by metal wedges (b) and locking keys (c)

can be regarded as the cumulative distribution function (CDF) of $Y$. This problem can be approached in different ways. A possible solution consists in the integration of the joint probability density function of the random variable vector. However, this approach is usually too complicated [3]. A possible alternative consists in the use of the Monte Carlo (MC) method, however this sampling procedure has the drawback of being computationally expensive and inefficient for high reliability devices.

Otherwise, numerical methods can be adopted. A detailed description of the evolution of these methods and of related advantages is contained in [2]. 
Most of them are based on the determination of a polynomial approximation of the relationship between $Y$ and the inputs. This approximation was initially determined in the neighbourhood of a specific point (MVFOSM): an important upgrade consisted in the development of the most probable point (MPP) methods. They performed the polynomial expansion at iteratively determined points, thus improving the accuracy of results. The most efficient numerical methods, FORM, AFOSM, and Advanced Mean Value (AMV) [3-4] can be regarded within this category.

\section{Subject}

A survey of the literature concerned with turbines and turbogenerators showed that very few practical applications of probabilistic analyses are described [5-6]. Moreover, even from the methodological point, the whole procedure for a reliability assessment, based on the experimental characterization of the involved materials, on the state of load and on the structural behaviour is generally not presented. From this point of view, interesting perspectives are contained in [2], where a linear reliability model is applied for the determination of the probability of failure of a turbogenerator rotor. In Refs. $[1-2,7]$ it is shown that the most suitable approach to the determination of the stochastic distributions of the involved input variables consists in the statistical processing of experimental data.

The AMV method is often cited as a suitable and powerful tool for probabilistic analyses [8], but a detailed description of the analytical steps to be followed as a general procedure is often missing. Another issue is related to the use of linear or higher order models: both can be analytically implemented and may have many applications. However, no paper deals with a comparison between the obtained results to investigate if the increasing complexity of the model has an impact on the accuracy of results.

The subject of the present paper consists in the determination of the probability of failure and of the safety index (having the meaning of a safety coefficient in the probabilistic environment) of the previously mentioned generator CRR. In particular, the present study focuses on the most serious failure mode of the CRR cracking in tension, when it swells under centrifugal force. The calculation is performed by combining experimental results [9-12], referring to the static, cyclic and fatigue behaviour of the involved material, to data regarding the local state of load at the CRR most loaded location. From the methodological point of view, full details are provided on the statistical and analytical models to be applied for the estimation of the CRR probability of failure at different stages of its life. In particular, it deals with the statistical processing of the experimental yields, the determination of 
the analytical model and finally with the computation of the safety index and of the failure probability CDF. The adoption of linear and quadratic models is investigated, analyzing the impact on the results, in terms of computational efficiency and accuracy.

\section{Material variables and distributions}

The most widely used material for CRR manufacturing is $18 \mathrm{Mn} 18 \mathrm{Cr}$. In the previous stage of the current research [9-12] it was experimentally characterized, by running static, cyclic and LCF tests on specimens machined from blanks of real CRRs, along two directions: radial and tangential. The choice of these directions was supported by previous experimental studies [13-14]. Moreover, it can be justified by considering the structural response of a thick ring loaded by an internal pressure: as an effect of this load, stresses along the two mentioned directions are generated.

In the present study, the curves referring to the tangential direction were considered for the computation of material parameters and of their distributions. This choice appeared to be the most reasonable, as this is the direction where the greatest principal stresses are generated. There was initial fear that the material could have an anisotropic behaviour and that the fatigue and reliability assessments may lead to different results depending on the direction considered for characterization. However, a statistical analysis performed in [10] showed that there were no significant differences between the curves determined along the two directions, in other words that the involved material had an isotropic behaviour. The Manson-Coffin curve was determined according to [15], by running 27 tests in the strain controlled mode, considering different strain amplitudes. The curve was determined by fitting procedures: the analytical procedure is summarized by the following equations, Eqs. (1-3). The subscripts $e l$. and $p l$. stand for strain amplitudes in the elastic and plastic fields, while $\sigma_{f}^{\prime}, \varepsilon_{f}^{\prime}, b, c$ are respectively the fatigue strength and ductility coefficients with related exponents. Considering Eq. (2) and Eq. (3), it is quite evident that both the elastic and the plastic curves are converted into straight lines in the logarithmic scale. In particular, regarding the elastic curve, $b$ and $\operatorname{Lg}\left(\sigma_{f}^{\prime}\right)$ have respectively the role of the slope and of the offset term, the same remark can be made for $c$ and $\operatorname{Lg}\left(\varepsilon_{f}^{\prime}\right)$, referring to the plastic curve. The nominal values of the aforementioned parameters were therefore easily determined [15] by linear interpolations of the experimental yields in the logarithmic scale.

$$
\begin{gathered}
\Delta \varepsilon / 2=\Delta \varepsilon_{e l .} / 2+\Delta \varepsilon_{p l .} / 2=\left(\sigma_{f}^{\prime} / E\right)(2 N)^{b}+\varepsilon_{f}^{\prime}(2 N)^{c} \\
\operatorname{Lg}\left(\Delta \varepsilon_{e l .} / 2\right)=\operatorname{Lg}\left(\sigma_{f}^{\prime} / E\right)+b \cdot \operatorname{Lg}(2 N)
\end{gathered}
$$




$$
\operatorname{Lg}\left(\Delta \varepsilon_{p l .} / 2\right)=\operatorname{Lg}\left(\varepsilon_{f}^{\prime}\right)+c \cdot \operatorname{Lg}(2 N)
$$

A simple statistical model, described in [16], was then adopted. This is a general-purpose model that makes it possible to determine the standard deviations of the slopes and of the offset terms of regression lines. The outcome is obviously based on the scatter of the data: a little scatter leads to low standard deviations, while a huge scatter reflects in larger deviation extents. The application of this model to the experimental data in [9-10], linearly interpolated by the Eqs. (2-3), resulted in the determination of the standard deviations to be applied to $\operatorname{Lg}\left(\sigma_{f}^{\prime}\right), \operatorname{Lg}\left(\varepsilon_{f}^{\prime}\right), b, c$. An important issue consists in the discussion on the probabilistic distributions to be considered for $\operatorname{Lg}\left(\sigma_{f}^{\prime}\right), \operatorname{Lg}\left(\varepsilon_{f}^{\prime}\right)$, $b, c$ and to be subsequently determined, based on the data. Regarding this question, there are sometimes different points of view in literature. In Refs. $[8,17] \log$-Normal distributions were assumed for $\sigma_{f}^{\prime}$ and $\varepsilon_{f}^{\prime}$ : this is the most used approach, confirmed also by more recent studies [2, 18-19], despite the analysis in [6], considering Normal distributions. Regarding the fatigue exponents, $b$ and $c$, their variability was sometimes neglected, e.g. in [8, 17], where they were regarded as deterministic constants. When considering their random variability, the Normal distribution is the most widely used, with applications in $[2,6,18-19]$. Consequently, following the most diffused approaches, Log-Normal distributions have been here considered for $\sigma_{f}^{\prime}$ and $\varepsilon_{f}^{\prime}$, and Normal distributions for the exponents $b$ and $c$. This assumption is the same as considering Normal distributions for $\operatorname{Lg}\left(\sigma_{f}^{\prime}\right), \operatorname{Lg}\left(\varepsilon_{f}^{\prime}\right), b$ and $c$ : these distributions are determined by the knowledge of the mean values, i.e. the aforementioned nominal values deriving from the regression model, and of the standard deviations. Thus, the combined application of the conventional linear regressions in the logarithmic scale [15], of the statistical model in [16] and of the most commonly accepted assumptions in literature can be regarded as a powerful tool for the conversion of LCF experimental data into statistical distributions. Finally, the elastic modulus $E$ in Eqs. (1-2) has been considered as a deterministic constant in agreement with several references, such as $[2,6,8,17-18]$. The determination of the coefficient distributions has two important implications. First of all, it is possible to determine confidence intervals to be applied to each material coefficient. From the graphical point of view, it is possible to determine lower (all the coefficients having their minimum value) and upper (max. values) bounds wrapped around the fatigue curve, accounting for the "worst scenario of twice the standard deviation". The internal band indicates the maximum likelihood states in the strain-life domain (Fig. 2). Secondly, the determination of stochastic distributions is the preliminary step for the reliability assessment of the studied component. The availability of input parameter distributions is in general a key issue of 
every reliability assessment. As reported, the described procedure makes it possible to easily determine reliable parameter distributions, supported by experimental data.

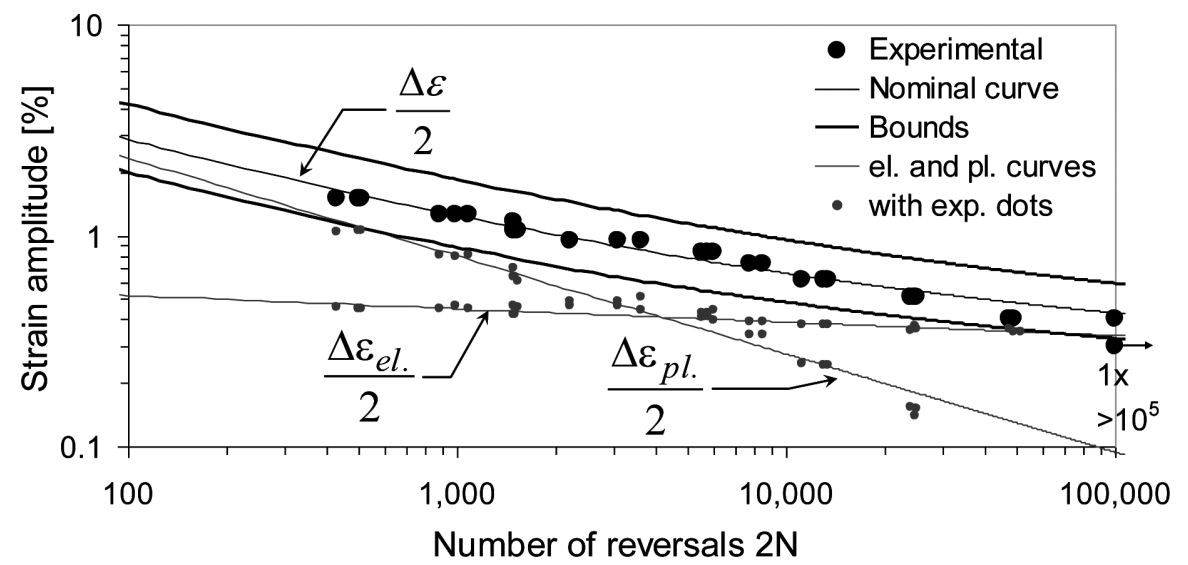

Fig. 2. LCF curve plotted together with its lower and upper bounds

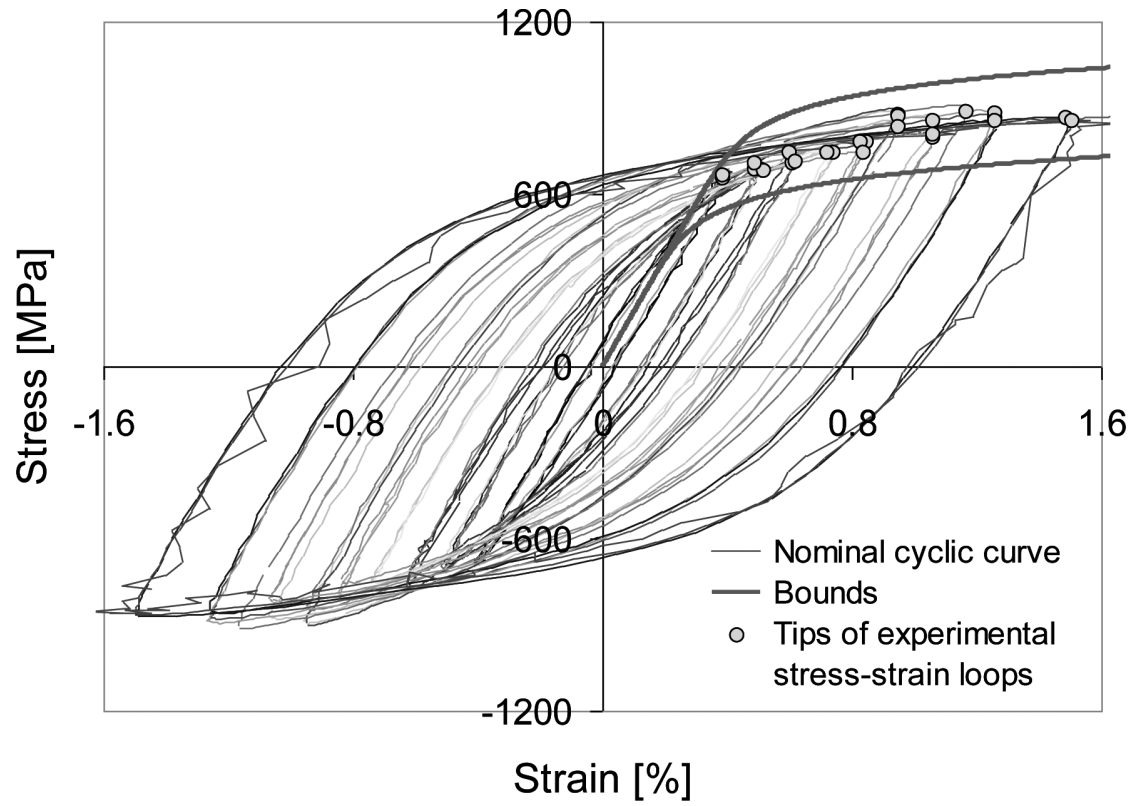

Fig. 3. Cyclic curve as an envelope of the tips of steady-state loops plotted together with its bounds in the plasticity related part

The static and cyclic curves were interpreted according to the RambergOsgood model: the related parameters, namely the Monotonic and the Cyclic Strength Coefficients $\left(K, K^{\prime}\right)$ and the related strain hardening components $\left(n, n^{\prime}\right)$ were again determined by fitting procedures in the Logarithmic scale. 
The applied analytical model is reported in Eqs. (4-5), with reference to the static curve. It can be observed that the function in Eq. 5 may be regarded as a straight line in the variables $\left[\operatorname{Lg}\left(\varepsilon_{p l}.\right), \operatorname{Lg}(\sigma)\right]$, where $n$ and $\operatorname{Lg}(K)$ have respectively the role of the slope and of the constant term. A similar relationship can be determined for the cyclic curve.

$$
\begin{gathered}
\varepsilon=\varepsilon_{e l .}+\varepsilon_{p l .}=\frac{\sigma}{E}+\left(\frac{\sigma}{K}\right)^{\frac{1}{n}} \\
\operatorname{Lg}\left(\varepsilon_{p l .}\right)=\frac{1}{n} \operatorname{Lg}(\sigma)-\frac{1}{n} \cdot \operatorname{Lg}(K) \Leftrightarrow \operatorname{Lg}(\sigma)=n \cdot \operatorname{Lg}\left(\varepsilon_{p l .}\right)+\operatorname{Lg}(K)
\end{gathered}
$$

The same steps described above, referring to the LCF curve, were followed when processing the experimental data of the static and cyclic curves [9-10]. The results were initially linearly interpolated as in Eq. (5), which led to the determination of the nominal values of $n, n^{\prime}, \operatorname{Lg}(K), \operatorname{Lg}\left(K^{\prime}\right)$. Afterwards, the statistical model in [16], was applied for the computation of the standard deviations of the slopes $n, n^{\prime}$ and of the constant terms $\operatorname{Lg}(K)$, $\operatorname{Lg}\left(K^{\prime}\right)$. In this case, also, the type of statistical distribution to be adopted for the determined parameters needs a discussion on the basis of literature. The most common approach [18-21] regards considering Log-Normal distributions for $K$ and $K^{\prime}$, i.e. Normal distributions for $\operatorname{Lg}(K)$ and $\operatorname{Lg}\left(K^{\prime}\right)$. Different options are available for the hardening exponents: sometimes they were considered as deterministic [8, 17], whereas in [18] they were presumed to be Log-normally distributed, and in [2, 20-21] Normal distributions were considered or estimated. In the present study, Log-Normal distributions have been considered for $K$ and $K^{\prime}$, and Normal distributions have been applied to the exponents $n$ and $n^{\prime}$, consistently with the modelling of the LCF curve parameters.

Like for the fatigue curve, the determination of the Normal distributions of the involved coefficients, made it possible to estimate confidence intervals and to determine lower and upper bounds enclosing the maximum likelihood stress-strain states. For instance, Fig. 3 shows the cyclic curve (determined as an envelope of the tip points of steady state stress-strain loops), together with its bounds accounting for the "worst scenario of twice the standard deviation". It can be observed that the bounds involve just the plasticity related region, as the Young's modulus is considered deterministic. Thus, in this case also, it was possible to determine statistical distributions, supported by experimental data, which are going to be processed as inputs for the CRR reliability assessment.

When considering the cyclic response of a material under LCF, an important issue concerns its behaviour, which is usually classified as Masing or non-Masing. The mathematical modelling of the structural response and 
life prevision is strictly related to this aspect. A Masing material exhibits ascending and descending branches of hysteresis loops being geometrically similar each other. The opposite behaviour, reflecting in dissimilar upward and downward hysteresis loop curves, is called non-Masing. These two responses are related the material micro-structure: when no dislocation cells form, even at high strains and loads, a Masing behaviour is usually observed. On the contrary, the frequent generation of dislocations leads to a non-Masing behaviour. It is possible to establish the material classification, by running the so-called Masing analysis, and producing the Master curve. It is obtained by plotting all of the hysteresis loops on a single diagram, so that all the inferior loop tips are made coincident in the origin: the curve is given by the envelope of the upward branches of the hysteresis loops. Afterwards, the Masing curve, i.e. the cyclic curve, magnified by a factor of two, must be plotted: its analytical expression is given by Eq. (6), where $\Delta \sigma$ and $\Delta \varepsilon$ are respectively the stress and the strain ranges, whereas, the other variables have the meaning exposed above.

$$
\Delta \varepsilon=\frac{\Delta \sigma}{E}+2 \cdot\left(\frac{\Delta \sigma}{2 K^{\prime}}\right)^{\frac{1}{n^{\prime}}}
$$

A Masing behaviour is indicated by the upward branches matching up each other, i.e. being theoretically overlapped and standing on the same Master curve [22-24]. This curve should be well interpolated by the Masing curve.

The here-described analysis has been performed, considering the experimental data in [9-10]: the determination of the Master curve, with superimposed Masing and nominal cyclic curves is depicted in Fig. 4. The here plotted stress-strain loops refer to the stabilized regions at about midlife and cover the entire strain amplitude range from $0.5 \%$ to $1.5 \%$. The material behaviour is undoubtedly Masing, as testified by the increasing loop branches well fitting each other and the Masing curve.

There was some concern regarding the possibility that the material may depart from the Masing model, when the accumulated damage due to the fatigue load becomes significant. In order to provide an answer to this question, the same analysis was repeated, considering experimental stress-strain hysteresis loops, recorded at about $80 \%$ of the observed full life. In particular, the here considered data were recorded at the following stages of the same trials that led to the loops in Fig. 4.

The result is shown in Fig. 5: it can be observed that the material behaviour is still Masing, as the upward branches are all overlapped. A reason for not departing from the Masing behaviour is surely related to the material response under fatigue. The experimental campaign in [9-10] showed that $18 \mathrm{Mn} 18 \mathrm{Cr}$ is able to maintain its stiffness properties almost until failure and 


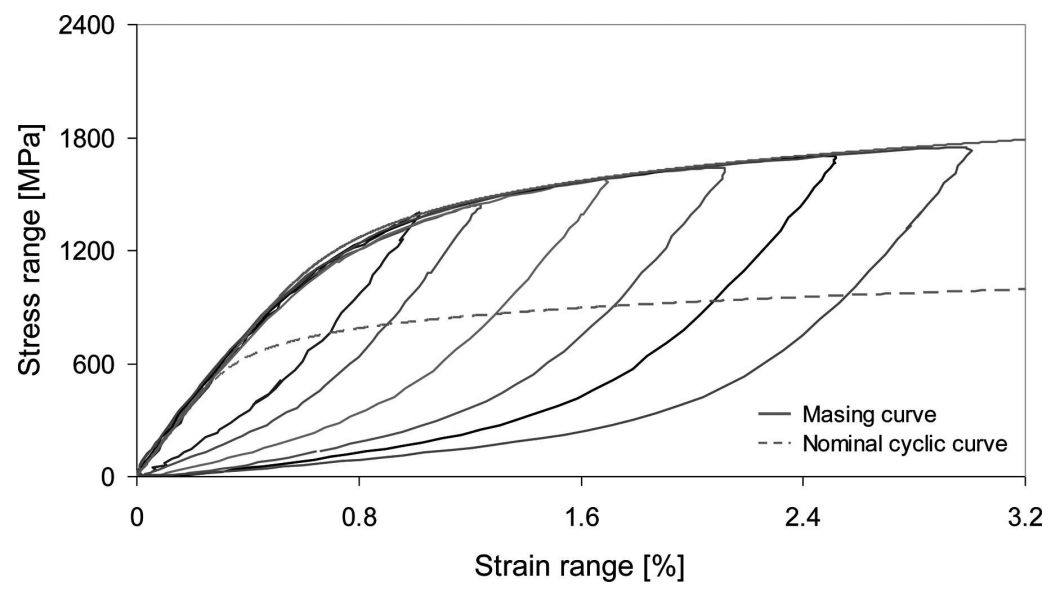

Fig. 4. Masing analysis of the experimental data in [9-10], considering stress-strain hysteresis loops at midlife

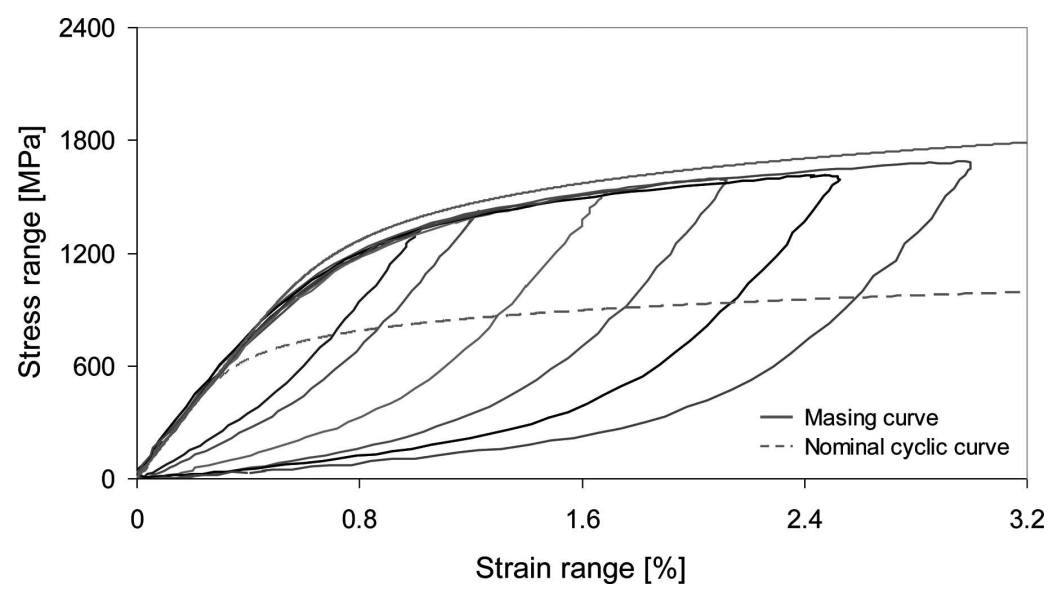

Fig. 5. Masing analysis of the experimental data in [9-10], considering stress-strain hysteresis loops at about $80 \%$ of full life

that the stabilized region for stress-strain loops is therefore much extended. In this region, the material exhibits a cyclic softening, with very low decrease for the stress at tip points. With respect to Fig. 4, a discrepancy can be observed between the Master curve and the Masing one. This mismatch is anyway quite low, in the order of few percentage points (up to 4\%), comparing stress range values at the same strain ranges. The reason for this discrepancy is indeed related to the just mentioned material cyclic softening.

The conclusion is that the material retains a Masing behaviour over a large extent of fatigue life and that the adopted cyclic stress-strain model is therefore reliable. 


\section{Mathematical models for the determination of local stress-strain loops and for life prediction}

The experimentally-determined material parameters make it possible to simulate the local stress-strain states at the most critical locations of the CRR. As mentioned above, the failure mode related to CRR swelling and cracking in tension was here considered, therefore the regions shown in Fig. 6 were considered for local analysis, as the most critical. This figure also shows sketches of the centrifugal forces transmitted by the coils to the CRRs and a scheme of the their distribution along the angular coordinate.

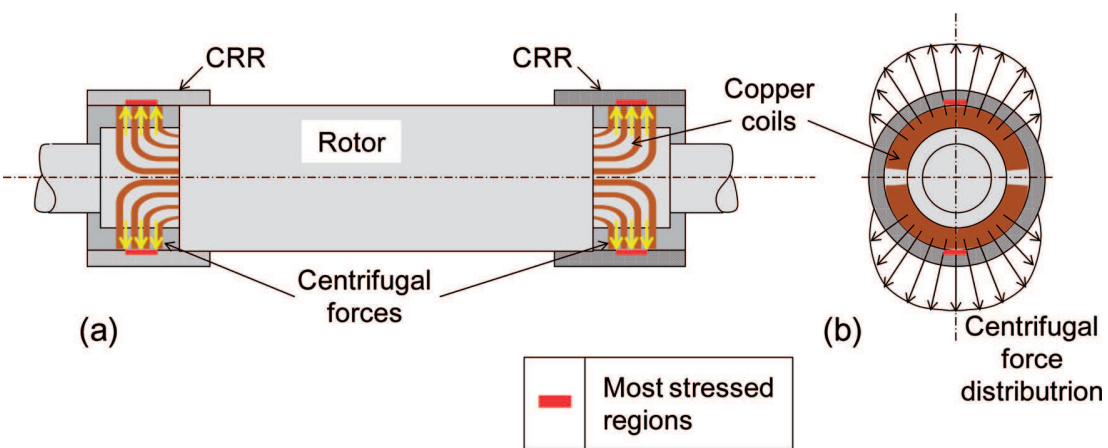

Fig. 6. CRRs connected to the rotor and their most stressed regions: front view (a) and lateral view with centrifugal force distribution (b)

The question was initially tackled for the deterministic point of view: the Neuber's hyperbola model was applied in the determination of tip points. The obtained loops, are qualitatively depicted in Fig. 7 and can be described as in the following:

- Curve 0-1: The CRR is shrunk-fitted onto the rotor over the coils: due to the contact between the internal part of the ring and the copper windings, the CRR is slightly loaded by an internal pressure. As a consequence a tangential tensile stress is generated.

- Cycle 1-2-3: machine pretesting at a $20 \%$ increased rotational speed $(3,600 \mathrm{rpm}$, point 2) and following stop (point 3). During rotation, the centrifugal force tends to eject the copper coils, which are restrained by the CRR: as a consequence, the CRR swells in tension and the tangential stress is much increased, thus overcoming the material yield strength. Afterwards, the speed is lowered down to zero upon machine switch off (point 3), the centrifugal force also drops to zero and the state of stress is therefore decreased.

- Cycle 3-4-5: Machine starting and speed increase up to the nominal value of 3,000 rpm (point 4); speed decrease down to zero, as the machine is 
switched off (point 5). This last cycle corresponds to the typical use of the turbogenerator, rotating at its nominal speed with about 10,000-15,000 transients, as explained in the Introductive Paragraph.

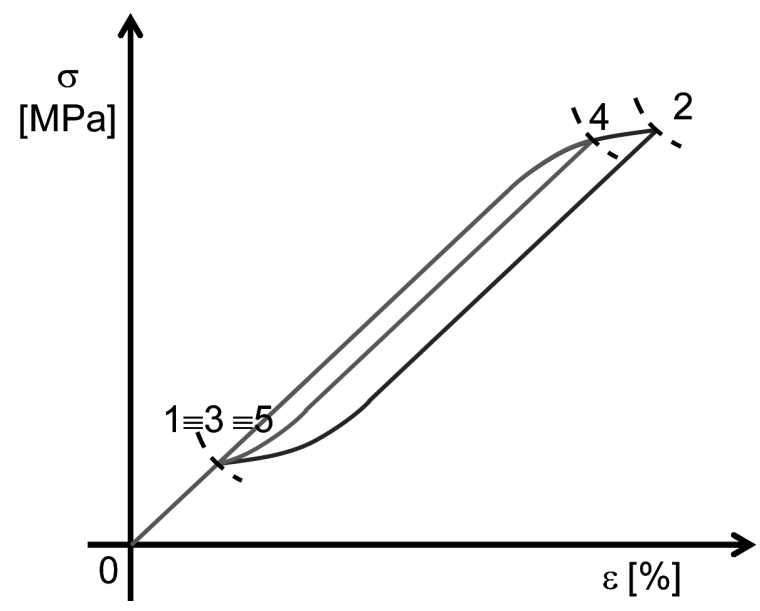

Fig. 7. Stress-Strain hysteresis loops during machine use

The first tip point can be determined by solving the two-equation system in Eq. (7), where the first equation refers to the analytical description of the static curve, while the second one stands for the Neuber's hyperbola. Similarly, the tip points of the loops are determined by solving the system in Eq. (8), where the term $\Delta$ indicates stress and strain ranges and the first equation is the analytical description of each hysteresis loop branch. This formula is based on the well known 2:1 homothetic relationship between the trend of hysteresis loop branches (whose envelope is the Master curve) and the shape of the cyclic curve for Masing materials. The adopted model in the plastic field presumes a kinematic hardening of the material. It must be argued that the stress and strain states are multi-axial, with components not only along the tangential, but also the radial direction.

$$
\begin{gathered}
\left\{\begin{array}{c}
\varepsilon=\frac{\sigma}{E}+\left(\frac{\sigma}{K}\right)^{\frac{1}{n}} \\
\sigma \cdot \varepsilon=\frac{\left(K_{T} \cdot S\right)^{2}}{E}=\sigma_{e l .} \cdot \varepsilon_{e l .}
\end{array}\right. \\
\left\{\begin{array}{l}
\frac{\Delta \varepsilon}{2}=\frac{\Delta \sigma}{2 E}+\left(\frac{\Delta \sigma}{2 K^{\prime}}\right)^{\frac{1}{n^{\prime}}} \\
\Delta \sigma \cdot \Delta \varepsilon=\frac{\left(K_{T} \cdot \Delta S\right)^{2}}{E}=\Delta \sigma_{e l .} \cdot \Delta \varepsilon_{e l .}
\end{array}\right.
\end{gathered}
$$

In order to extend Eqs. (7-8) to the multi-axial stress condition, the equivalent criterion of Von Mises stress and strain is adopted here, so equivalent 
Von Mises stress and strain amplitudes must be considered. It can be justified by observing that all the stress-strain components experience in phase cyclic variations; moreover, it is a common approximation in the study of the LCF performance of bodies extended in the three dimensions [6, 25].

The stress concentration coefficient, $K_{T}$, and the theoretical stresses $(S$, $\Delta S$ ) in the second equations, cannot be correctly defined for not-beam components, however the constant term of the hyperbolic distribution can be easily estimated by the computation of the stress and the strain $\left(\sigma_{e l .}\right.$ and $\varepsilon_{e l .}$, Eq. (7)) or of their ranges $\left(\Delta \sigma_{e l}\right.$. and $\Delta \varepsilon_{e l .}$, Eq. (8)), considering a linear elastic model. They can be regarded as the "nominal state of load" and can be easily computed, by running FEM simulations in the elastic field, considering the limit conditions of switched off machine (zero rotational speed), of rotor rotating at the speeds of $3,600 \mathrm{rpm}$ in the pre-trial conditions and of 3,000 in the conventional use.

Finally, the last issue, concerning life estimation, is tackled using the experimentally-determined fatigue curve. The Manson - Coffin strain-life model is usually applied for structures under LCF. Its conventional formulation, reported in Eq. (1) can be modified, as in Eq. (9), to take the effects of mean stress $\sigma_{m}$ and mean strain $\varepsilon_{m}$ on the fatigue life into consideration [26]. In the present case the effect of the mean stress on the elastic part of the curve was considered only. The impact of the mean strain was reasonably presumed to be negligible, as $\varepsilon_{m}$ is about two orders of magnitude lower than $\varepsilon_{f}^{\prime}$, also in agreement with [23, 27]. The Manson-Coffin model in its modified Morrow formulation was therefore utilized for closed loop life estimation. From the operative point of view, life estimation up to crack initiation is conducted by applying Eq. (9) to the two closed loops (cycles 1-2-3 and 3-4-5) in Fig. 7 and by the following application of a cumulative damage theory (the Palmgren-Miner model).

$$
\frac{\Delta \varepsilon}{2}=\frac{\sigma_{f}^{\prime}-\sigma_{m}}{E}(2 N)^{b}+\left(\varepsilon_{f}^{\prime}-\varepsilon_{m}\right)(2 N)^{c} \cong \frac{\sigma_{f}^{\prime}-\sigma_{m}}{E}(2 N)^{b}+\varepsilon_{f}^{\prime}(2 N)^{c}
$$

It could be argued that the stress-strain hysteresis loops during machine use are not symmetric (Fig. 7). In this loading condition, the "Ratcheting effect" (or cyclic creep) may occur, due to plastic strain accumulation. This effect takes place in uniaxial loading with a non-zero mean stress. The material behaviour is different, depending if load is applied in stress or in strain controlled condition.

In the first case, plastic strain accumulation takes place and the mean strain starts to increase cycle by cycle. Three different responses may be expected: (1) after a few tens of cycles the material behaviour becomes purely elastic ("adaptation"), (2) the material keeps an elasto-plastic behaviour, but 
the ratcheting rate (i.e. the rate of mean strain increase) rapidly drops to zero ("accommodation"), (3) the mean strain keeps increasing and constant ratcheting takes place [28-29].

When cycling in the strain-controlled mode, the value of mean stress decreases to zero, due to stress relaxation, with the result that the initial absence of symmetry tends to disappear cycle by cycle. [28-29]. For this reason, the ratcheting effect, as plastic strain accumulation, is mainly referred to stress controlled rather than to strain controlled conditions [30-32].

As stated in [33], ratcheting response highly depends on temperature, material micro-structural characteristics and on the type of load.

Regarding the first issue, it must be remarked that the CRR is not subjected to elevated temperatures during the machine use: the operating maximum temperature is about $70^{\circ}$ [10] and is therefore not much higher than room temperature.

With reference to material characteristics, studies concerning the ratcheting effect on $18 \mathrm{Mn} 18 \mathrm{Cr}$ have never been reported in literature, however, some results are available regarding austenitic stainless steels (like the studied material). A material with these properties was asymmetrically loaded in stress controlled condition in Ref. [29], and the experimentation was conducted at three temperatures (room temperature, $300^{\circ} \mathrm{C}, 700^{\circ} \mathrm{C}$ ). It was shown that at room temperature the ratcheting rate gradually decreases after few tens of cycles, when "accommodation" occurs. This result is confirmed by related references.

Finally, regarding the type of load, it must be argued that the loading mode at the most stressed locations of the CRR is closer to strain controlled rather than to stress controlled conditions. The reason is that just small material volumes in these regions are involved by plastic deformations. The process of plastic deformation is therefore constrained and limited by the surrounding material that maintains an elastic behaviour. This is in agreement with common assumptions governing LCF.

As a matter of the fact, the loops in Fig. 7 are expected to experience a mean stress relaxation during cycling and, as a secondary effect, a slight increase of the mean strain. Regarding the first issue, the proposed model, accounting for mean stress without considering relaxation, yields conservative results. Referring to the second issue, the mean strain increase is expected to stop after few tens of cycles, as "accommodation" occurs.

For the aforementioned reasons it seemed to be reasonable to neglect the ratcheting effect in the previously detailed analytical modelling of the material cyclic behaviour. 


\section{Resume of the input variables for the probabilistic study}

The question of the simulation of the stress-strain hysteresis loops and of life prediction was tackled from the probabilistic point of view with the adoption of the just described models. The first step of the reliability assessment consisted in the discussion on the variables to be considered in the reliability assessment. In the previous paragraphs it was shown how to determine the stochastic properties of the material the CRR is made of, at this stage the discussion involved the opportunity of also regarding the "state of load" as a random variable. For "state of load", the parameters regarding the local states of elastic stress and strain at the most stressed CRR location $\left(\sigma_{e l .}, \varepsilon_{e l .}, \Delta \sigma_{e l .}\right.$, $\Delta \varepsilon_{e l .}$ ) must be intended. On this issue it must be observed that the CRR is shrunk-fitted onto the rotor according to a stiff and careful procedure, where the thermal cycle of the CRR is carefully optimized, based on the entity of interference, the coefficient of thermal expansion of the material, the necessity of preventing any damage to the coils. As a consequence, the tolerance specifics of the rotor and of the CRR are very strict and subjected to a severe quality control, which makes sure of the repeatability of the coupling procedure. During machine use, the rotor spins at the speed of 3,600 rpm in the pre-trial and at the speed of 3,000 in nominal conclusions. The rotational speed is strictly controlled, as it must be maintained at a fixed constant value during electric energy generation. As a consequence, the centrifugal force has a controlled value and it can be excluded that it experiences fluctuations during rotation. For the listed reasons, it was finally decided to regard the "state of load" as a deterministic parameter. This choice was also inspired by some interesting remarks contained in [34]. This study regards a strainbased fatigue reliability analysis, involving a nuclear reactor material. The motivation of this research consisted in the experimental observation of a significant scatter of the cyclic stress-strain responses of the studied material. In particular, it was highlighted that:

- Without considering this scatter, a non-conservative evaluation might be given.

- It is important to take the aforementioned scatter into account, as a random cyclic strain history can be introduced even under a deterministic loading history. In other words, more focus must be given on the modelling of the scatter in the cyclic behaviour of the material, rather than on the modelling of the random variations of the applied load.

The CRR material also exhibits a not negligible cyclic scatter (Fig. 3) and a (lower) static scatter. It was consequently decided to consider the slopes $\left(n, n^{\prime}\right)$ and the constant terms $\left(\operatorname{Lg}(K), \operatorname{Lg}\left(K^{\prime}\right)\right)$ of both the static and cyclic curves as random variables with Normal distributions $\left(U_{1}, \ldots, U_{4}\right)$. In the 
"Material variables and distributions" paragraph, the proper choice of Normal distributions was discussed and justified. The distribution parameters were determined, following the described method, based on experimental data [910]. According to [34], this approach makes it possible to account for the random properties of the local stress-strain states in the elastic-plastic field, despite the approximation of deterministic loading history.

Other four random variables $\left(U_{5}, \ldots, U_{8}\right)$ were associated to the coefficients of the Manson-Coffin model: Normal distributions were presumed for respectively $\operatorname{Lg}\left(\sigma_{f}^{\prime}\right), \operatorname{Lg}\left(\varepsilon_{f}^{\prime}\right), b, c$, in agreement with the previously reported literature review. The above described procedure was followed for the determination of distribution parameters, on the basis of the experimental data in [9-10]. The final remark regards the choice of the output variable. The goal of the probabilistic analysis consisted in the determination of the CDF of the expected life $N$. Consequently, the device life was chosen as the output variable $Y$. However, as suggested in [8, 17, 20, 34], a logarithmic scale was adopted, since Log-Normal distribution is the most suitable for describing the statistical scattering of the fatigue life. Therefore, $Y=\operatorname{Lg}(N)$ was considered in the analytical computation according to the procedure described below. As previously remarked, the Young's Modulus, was considered as a deterministic constant $[2,6,8,17-18]$, with the experimentally determined value of 193.8 GPa [9-10].

A resume of the input and output variables is shown in Tab. 1: based on the previous observations, all the variables have Normal distributions, defined by the related mean values (i.e. nominal values resulting by the regression procedure, $\mu$ ) and standard deviations (STD).

\section{Numerical Procedure}

The expected life is indeed related to the input variables and to the "state of load": as explained above, its computation requires the simulation of the static curve and of the steady-state hysteresis loops and the application of a strain-life model, with final processing of results by a cumulative damage theory. Two remarks can be made on this procedure: the involved equations are not linear and do not admit a closed form analytical solution. In other words, it is not possible to find the analytical expression of the functional relationship $g$ between the input variables and life.

The AMV utilizes a Taylor expansion to achieve a suitable approximation of the function $g$ in the neighbourhood of a most probable point (MPP). The MPP is usually referenced as the "design point": it is defined in the multidimensional space of the random variables and its coordinates indicate the values of the input variables, which are likely to lead to a failure and are at 
RELIABILITY ASSESSMENT OF A TURBOGENERATOR COIL RETAINING RING BASED. .

Table 1.

Input and output random variables with statistical data

\begin{tabular}{|c|c|c|}
\hline \multicolumn{3}{|c|}{ Input random variables } \\
\hline Variable & Mean value $(\mu)$ & Standard Deviation $(S T D)$ \\
\hline $\operatorname{Lg}\left(\sigma_{f}^{\prime}\right)=U_{1}$ & 3.120 & 0.019 \\
\hline $\operatorname{Lg}\left(\varepsilon_{f}^{\prime}\right)=U_{2}$ & -0.701 & 0.059 \\
\hline$b=U_{3}$ & -0.063 & 0.005 \\
\hline$c=U_{4}$ & -0.465 & 0.016 \\
\hline$n=U_{5}$ & 0.008 & $3 \cdot 10^{-5}$ \\
\hline $\operatorname{Lg}(K)=U_{6}$ & 3.112 & $4 \cdot 10^{-5}$ \\
\hline$n^{\prime}=U_{7}$ & 0.098 & 0.009 \\
\hline $\operatorname{Lg}\left(K^{\prime}\right)=U_{8}$ & 3.131 & 0.023 \\
\hline \multicolumn{3}{|c|}{ Output random variable } \\
\hline \multicolumn{3}{|c|}{$Y=\operatorname{Lg}(N)$} \\
\hline
\end{tabular}

the same time the closest to the mean values of the variables. In this case, it represents the values of material parameters that imply a premature failure and are at the same time as close at possible to the nominal values. The computational difficulties arise from the occurrence that the design point is initially unknown. Consequently, it must be determined only by an iterative method: the first expanding point for the Taylor expansion is set as the point defined by the mean values of the random variables. Let $\underline{a}=\left[a_{1}, \ldots, a_{r}\right]^{T}$ be the expanding point. For the sake of generality, $r$ random variables are considered $\left[U_{1}, \ldots, U_{r}\right]^{T}$, with $r=8$ in the case of the present study.

Different approaches can be used in the determination of the polynomial expression of $g$ by Taylor expansion: linear and not linear (e.g., second-order models). Linear models are usually applied [2, 8, 17], but it could be argued that the relationship between input and output variables is indeed not linear. In the present paper, both linear and second-order formulations have been considered, the linear approach is described in the following lines.

$$
\begin{aligned}
& Y\left(U_{1}, \ldots, U_{r}\right)=\operatorname{Lg}(N)= \\
& =g(\underline{a})+\left.\frac{\partial g}{\partial U_{1}}\right|_{(\underline{a})}\left(U_{1}-a_{1}\right)+\ldots+\left.\frac{\partial g}{\partial U_{i}}\right|_{(\underline{a})}\left(U_{i}-a_{i}\right)+\ldots+\left.\frac{\partial g}{\partial U_{r}}\right|_{(\underline{a})}\left(U_{r}-a_{r}\right)+(H . O . T .)= \\
& =g(\underline{a})+\sum_{i=1}^{r}\left[\left.\frac{\partial g}{\partial U_{i}}\right|_{(\underline{a})}\left(U_{i}-a_{i}\right)\right]= \\
& =\alpha_{0}+\alpha_{1}\left(U_{1}-a_{1}\right)+\ldots+\alpha_{i}\left(U_{i}-a_{i}\right)+\ldots+\alpha_{r}\left(U_{r}-a_{r}\right)
\end{aligned}
$$

The first-order expansion around the point $\underline{a}$ is reported in Eq. (10), where the term "H.O.T." indicates neglected terms. The derivative terms may be 
easily numerically calculated by the finite difference methods. In particular, the partial derivatives are approximated by quotients of finite differences determined by operating slight perturbations of the random variables around their values at the expanding point. Eq. (11) shows the computation of the (i-th) derivative term, by the forward finite difference approach [3].

$$
\left.\frac{\partial g}{\partial U_{i}}\right|_{(\underline{a})} \approx \frac{\Delta g}{\Delta U_{i}}=\frac{g\left(a_{1}, \ldots, a_{i}+\Delta U_{i}, \ldots, a_{n}\right)-g\left(a_{1}, \ldots, a_{i}, \ldots, a_{n}\right)}{\phi_{i}+\Delta U_{i}-\phi_{i}}
$$

The term $\Delta U_{i}$ in Eq. (11) represents the entity of the (i-th) perturbation: it must be a good compromise from being too high (would imply a rough estimation of the partial derivative punctual value) and being too low (would imply a very stiff polynomial approximation, being reliable just in a small surrounding of the expanding point). For this purpose, Refs. [8, 17] suggest to choose the perturbation size $\Delta U_{i}$ as the $10 \%$ of the (i-th) variable standard deviation, i.e. $\Delta U_{i}=0,1 \cdot S T D\left(U_{i}\right)$. From the operative point of view, the constant term and the derivative ones were determined, by running the mathematical model described above for nine times. A linear model requires in general $(r+1)$ estimations for the computation of the $\alpha_{i}$ coefficients in Eq. (10) [8].

Let us consider a second-order model. Its expression, determined as a second-order Taylor expansion of a multi-variable function, can be written as follows, Eq. (12):

$$
\begin{aligned}
& Y\left(U_{1}, \ldots, U_{r}\right)=\operatorname{Lg}(N)= \\
& =g(\underline{a})+\left.\frac{\partial g}{\partial U_{1}}\right|_{(\underline{a})}\left(U_{1}-a_{1}\right)+\ldots+\left.\frac{\partial g}{\partial U_{i}}\right|_{(\underline{a})}\left(U_{i}-a_{i}\right)+\ldots+\left.\frac{\partial g}{\partial U_{r}}\right|_{(\underline{a})}\left(U_{r}-a_{r}\right)+ \\
& +\left.\frac{1}{2} \frac{\partial^{2} g}{\partial U_{1}^{2}}\right|_{(\underline{a})}\left(U_{1}-a_{1}\right)^{2}+\ldots+\left.\frac{1}{2} \frac{\partial^{2} g}{\partial U_{i}^{2}}\right|_{(\underline{a})}\left(U_{i}-a_{i}\right)^{2}+\ldots+\left.\frac{1}{2} \frac{\partial^{2} g}{\partial U_{r}^{2}}\right|_{(\underline{a})}\left(U_{r}-a_{r}\right)^{2}+ \\
& +\left.2 \sum_{i=1}^{r-1} \sum_{j=i+1}^{r} \frac{\partial^{2} g}{\partial U_{i} \partial U_{j}}\right|_{(\underline{a})}\left(U_{i}-a_{i}\right)\left(U_{j}-a_{j}\right)+H . O . T .= \\
& =g(\underline{a})+\sum_{i=1}^{r}\left[\left.\frac{\partial g}{\partial U_{i}}\right|_{(\underline{a})}\left(U_{i}-a_{i}\right)\right]+\frac{1}{2} \sum_{i=1}^{r}\left[\left.\frac{\partial^{2} g}{\partial U_{i}^{2}}\right|_{(\underline{a})}\left(U_{i}-a_{i}\right)^{2}\right]+ \\
& +\left.2 \sum_{i=1}^{r-1} \sum_{j=i+1}^{r} \frac{\partial^{2} g}{\partial U_{i} \partial U_{j}}\right|_{(\underline{a})}\left(U_{i}-a_{i}\right)\left(U_{j}-a_{j}\right) \cong \\
& \cong \alpha_{0}+\alpha_{1}\left(U_{1}-a_{1}\right)+\ldots+\alpha_{i}\left(U_{i}-a_{i}\right)+\ldots+\alpha_{r}\left(U_{r}-a_{r}\right)+ \\
& +\alpha_{r+1}\left(U_{1}-a_{1}\right)^{2}+\ldots+\alpha_{r+i}\left(U_{i}-a_{i}\right)^{2}+\ldots+\alpha_{2 r}\left(U_{r}-a_{r}\right)^{2}
\end{aligned}
$$

It can be observed that the polynomial expression in its compact form consists of four terms: the first and the second are the same of the first order 
model, while the third and the fourth ones contain higher order derivatives. In particular, the last one contains mixed derivatives ad is usually regarded as the "mixed term". In Ref. [8] it is remarked that the minimum data set to achieve the computation of all the derivative terms is $(r+2)(r+1) / 2$, when considering all the terms in Eq. (12). The dimension of the data set is lowered to $(2 r+1)$ when the mixed term is neglected. In the present study, 72 function evaluations would be required in the first case and 17 in the second one. In Ref. [8] it is therefore suggested to neglect the last term. The provided reasons obviously involve the simplicity of the model and the efficiency in its practical application. In addition, it is emphasized that, in the polynomial approximation of highly not linear relationships, problems of numerical ill-conditioning may arise, reducing the accuracy of the Taylor expansion. The mixed term was therefore cut and the final expression at the end of Eq. (12) was used for practical applications. The first step consisted in the estimation of the $(2 r+1)$ constant terms, indicated as $\alpha_{0}, \ldots, \alpha_{2 r}$. They can be determined by an interpolation procedure that requires $(2 r+1)$ function evaluations: in particular, the CRR life was computed for $(2 r+1)$ different data sets, applying the complete mathematical model. According to [8], the data sets were chosen as in Table 2: the calculations led to the estimations of the output variables $Y_{0}, \ldots, Y_{2 r}$, computed as base 10 logarithms of the estimated lives. The $\Delta U_{i}$ terms retain the same meaning of slight perturbations of the random variables around the related expanding points.

Table 2.

Data sets for the computation of $\alpha_{i}$ constant terms

\begin{tabular}{|c|c|c|c|c|c|}
\hline$U_{1}$ & $\ldots$ & $U_{i}$ & $\ldots$ & $U_{r}$ & $Y$ \\
\hline$a_{1}$ & $\ldots$ & $a_{i}$ & $\ldots$ & $a_{r}$ & $(Y)_{0}$ \\
\hline$a_{1}+\Delta U_{1}$ & $\ldots$ & $a_{i}$ & $\ldots$ & $a_{r}$ & $(Y)_{1}$ \\
\hline$a_{1}-\Delta U_{1}$ & $\ldots$ & $a_{i}$ & $\ldots$ & $a_{r}$ & $(Y)_{2}$ \\
\hline$\ldots$ & $\ldots$ & $\ldots$ & $\ldots$ & $\ldots$ & $\ldots$ \\
\hline$a_{1}$ & $\ldots$ & $a_{i}+\Delta U_{i}$ & $\ldots$ & $a_{r}$ & $(Y)_{2 i-1}$ \\
\hline$a_{1}$ & $\ldots$ & $a_{i}-\Delta U_{i}$ & $\ldots$ & $a_{r}$ & $(Y)_{2 i}$ \\
\hline$\ldots$ & $\ldots$ & $\ldots$ & $\ldots$ & $\ldots$ & $\ldots$ \\
\hline$a_{1}$ & $\ldots$ & $a_{i}$ & $\ldots$ & $a_{r}+\Delta U_{r}$ & $(Y)_{2 r-1}$ \\
\hline$a_{1}$ & $\ldots$ & $a_{i}$ & $\ldots$ & $a_{r}-\Delta U_{r}$ & $(Y)_{2 r}$ \\
\hline
\end{tabular}

The constant terms were finally determined by solving the $(2 r+1)$ equation linear system in Eq. (13). 


$$
\left\{\begin{array}{l}
\alpha_{0}=(Y)_{0} \\
\alpha_{0}+\alpha_{1} \cdot \Delta U_{1}+\alpha_{r+1} \cdot\left(\Delta U_{1}\right)^{2}=(Y)_{1} \\
\alpha_{0}-\alpha_{1} \cdot \Delta U_{1}+\alpha_{r+1} \cdot\left(-\Delta U_{1}\right)^{2}=(Y)_{2} \\
\ldots \\
\alpha_{0}+\alpha_{i} \cdot \Delta U_{i}+\alpha_{r+i} \cdot\left(\Delta U_{i}\right)^{2}=(Y)_{2 i-1} \\
\alpha_{0}-\alpha_{i} \cdot \Delta U_{i}+\alpha_{r+i} \cdot\left(-\Delta U_{i}\right)^{2}=(Y)_{2 i} \\
\ldots \\
\alpha_{0}+\alpha_{r} \cdot \Delta U_{r}+\alpha_{2 r} \cdot\left(\Delta U_{r}\right)^{2}=(Y)_{2 r-1} \\
\alpha_{0}-\alpha_{r} \cdot \Delta U_{r}+\alpha_{2 r} \cdot\left(-\Delta U_{r}\right)^{2}=(Y)_{2 r}
\end{array}\right.
$$

The rest of the numerical procedure is the same for both linear and second-order models. The following step consisted in the analytical formulation of the failure function $h$, Eq. (14). It required the choice of a reference value $y=\operatorname{Lg}\left(N_{0}\right)$, where $N_{0}$ stands for the number of cycles for which the probability of failure is being computed and can be associated to the life mission. The condition $\operatorname{Lg}(N)=Y=y=\operatorname{Lg}\left(N_{0}\right)$ represents the limit state at the boundary between failure and service.

$$
\begin{aligned}
& h\left(U_{1}, \ldots, U_{r}\right)=Y\left(U_{1}, \ldots, U_{r}\right)-y= \\
& =\alpha_{0}+\alpha_{1}\left(U_{1}-a_{1}\right)+\ldots+\alpha_{i}\left(U_{i}-a_{i}\right)+\ldots+\alpha_{r}\left(U_{r}-a_{r}\right)-y=0
\end{aligned}
$$

It can be observed that a positive value of $h$ indicates that the computed life is greater than its reference value (e.g., set by design specifics), which implies a safety condition. Whereas, the condition $h<0$ has the physical meaning of the computed life being lower than the required mission for the turbogenerator rotor, in other words a failure must be expected during turbogenerator use. Finally, as previously stated, the condition of $h=0$, indicates the limit state, where the computed life is exactly equal to the required duration.

The further step consisted in variable reduction, i.e. the transformation of the basic variables into the reduced ones. The reduced variables exhibit a Normal distribution with a mean of zero and standard deviation normalized to one. This procedure was performed according to Eq. (15), where $U_{i}$ and $u_{i}$ respectively indicate the $(\mathrm{i}-\mathrm{th})$ random variable and the corresponding reduced one.

$$
u_{i}=\frac{U_{i}-\mu\left(U_{i}\right)}{S T D\left(U_{i}\right)} \Leftrightarrow U_{i}=u_{i} \cdot S T D\left(U_{i}\right)+\mu\left(U_{i}\right)
$$

All the basic variables in Eq. (14) were thus replaced by the related reduced coordinates. This computational procedure may be regarded as a 
change of the reference system: in the new one the point defined by the mean (i.e. nominal) values of all the input variables is moved to the origin.

Finally, the last step led to the determination of the design point and of its distance from the origin of the new reference system in the reduced variables: this distance is called safety index, $\beta$. Computation was performed by solving the following constrained optimization problem, Eq. (16).

$$
\left\{\begin{array}{l}
h\left(\underline{u}^{*}\right)=0 \\
\beta=\min \left\|\underline{u}^{*}\right\|
\end{array}\right.
$$

The symbol * in Eq. (16) indicates the coordinates at the design point: the equations in the constrained system have the following meaning. The design point is univocally determined as the point lying on the failure function (i.e. the limit state between safe and unsafe conditions) that is at the minimum distance from the origin of the reduced reference system. A graphical interpretation of the failure function separating the safe and unsafe domains, of the meanings of the design point and the safety index is shown in Fig. 8. For the sake of clarity, the graphical representation is sketched in a bidimensional reference system $\left(u_{i}-u_{j}\right)$, while, in reality, the failure function $h$ should be plotted in a $r$-dimensional space. From the computational point of view, the constrained system was easily solved by the Lagrange multipliers method, by the application of the following equations, Eqs. (17-18).

$$
\begin{gathered}
\lambda_{i}=\frac{\left.\frac{\partial h}{\partial u_{i}}\right|_{\left(\frac{a_{1}-\mu\left(U_{1}\right)}{\operatorname{STD}\left(U_{1}\right)}, \ldots, \frac{a_{n}-\mu\left(U_{n}\right)}{\operatorname{STD}\left(U_{n}\right)}\right)}}{\sqrt{\sum_{j=1}^{n}\left(\left.\frac{\partial h}{\partial u_{j}}\right|_{\left.\left(\frac{a_{1}-\mu\left(U_{1}\right)}{\operatorname{STD}\left(U_{1}\right)}, \ldots, \frac{a_{n}-\mu\left(U_{n}\right)}{\operatorname{STD}\left(U_{n}\right)}\right)\right)^{2}}\right.}} \\
\underline{u}^{*}=\beta \cdot\left[\lambda_{1}, \ldots, \lambda_{i}, \ldots, \lambda_{n}\right]^{T}
\end{gathered}
$$

In Eq. (17), the partial derivatives of the failure function $h$ are calculated at the expanding point, here expressed in the reduced coordinates. Its application makes it possible to compute the terms indicated by $\lambda_{i}$, which are the direction cosines of a vector that defines the design point. Subsequently, by applying the relationship in Eq. (18), considering the first relationship of Eq. (16), it is possible to estimate the safety index $\beta$ and to determine the reduced coordinates of the design point.

The probability of failure $p_{f}$ is finally calculated by the formula in Eq. (19) [8], where the symbol $\phi$ stands for the Standard Normal CDF (see also Fig. 8).

$$
p_{f}=\phi(-\beta)
$$




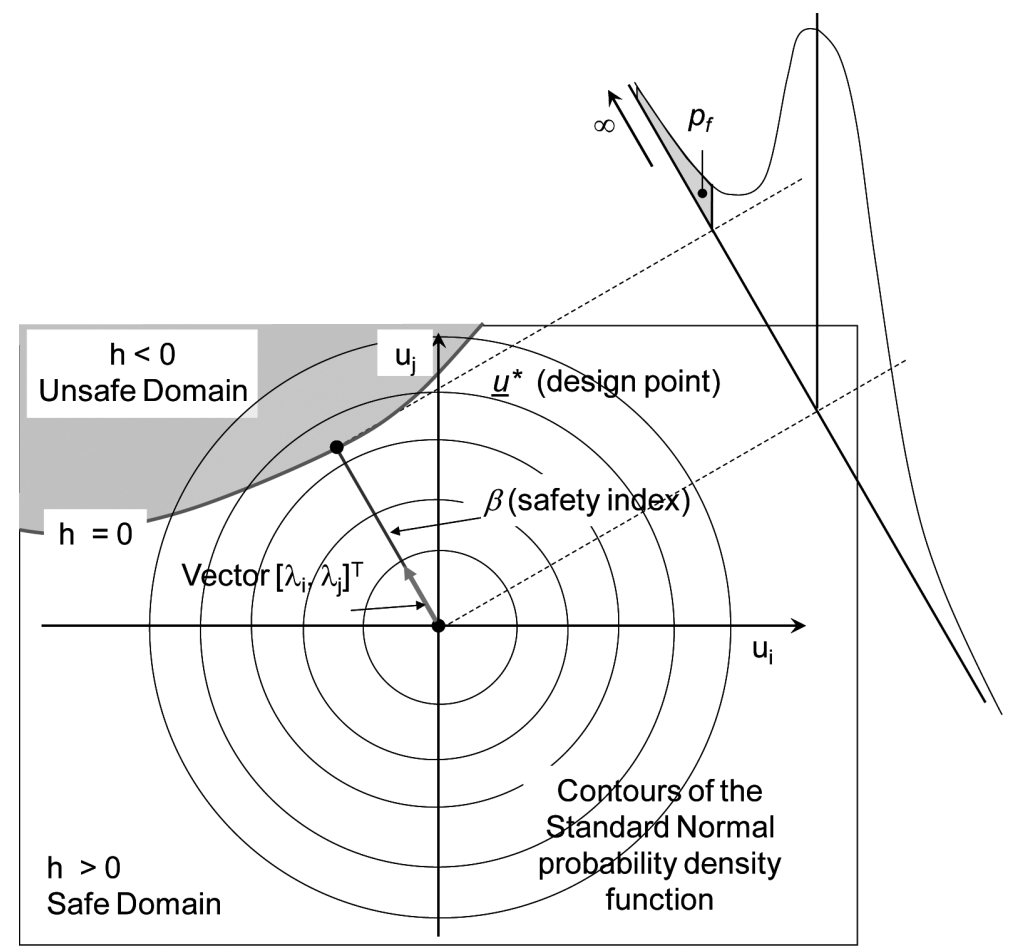

Fig. 8. Graphical determination of the safety index, the design point and the probability of failure in a two dimensional reduced reference system

The determined solution is usually regarded as the "Mean value (MV) solution" [4] and is frequently inaccurate. It can be explained by observing that the result depends on the determined coordinates of the design point, by Eqs. (16-18). All the computation process is based on the calculation of the firstor second-order polynomial expression relating life to the input variables. As previously stated, this expression is an expansion in the neighbourhood of the expanding point. In theory, the expansion should be performed at the design point or in the surroundings of it. However, as remarked at the beginning of this paragraph, the design point is not known a priori, therefore, the first expanding point is arbitrary set and may be even far away from the design point. This expansion at an arbitrary point is the main reason for the first polynomial expansion being often a poor approximation of the life inputs relationship at the design point. This problem can be overcome by an iterative procedure. A new expanding point is therefore set at the just determined design point and a different Taylor expansion is computed. The same algorithm is then repeated for the determination of a new design point. At the following step, a new expanding point is set at the just computed 
design point, ... Iterations are repeated until convergence. Few of them are usually required: in the present case just three were sufficient.

In order to determine the whole $\mathrm{CDF}$, in the life duration of the studied machine, different values of $N_{0}$ were considered, in a range up to 100,000 cycles. The computational procedure described above was therefore repeated for different values of $N_{0}$ in the specified range. The computation of the $\mathrm{CDF}$ of the rotor life was completed by an additional Monte Carlo (MC) simulation. It was performed at a high number of samplings, $10^{6}$, and its results were compared to the previously obtained yields, in order to make a comparative analysis, also in terms of computational efficiency. The firstand second-order numerical algorithms for the iterative calculation of the probability of failure and the MC simulation were implemented by writing custom routines in the Matlab environment (release 7.9.0.529 (R2009b)).

\section{Results and Discussion}

Both the models, the linear and the non-linear one, were applied for the reliability assessment of the turbogenerator CRR and led to the computation of the safety index and of the probability of failure, to be compared to literature reference values. The simultaneous application of both the approaches was a good opportunity to compare their responses in terms of efficiency and accuracy of the results. In a previous paper by the same author [2] a linear model had been developed and applied to the probabilistic analysis of a turbogenerator rotor, at its shrink-fit region. The obtained results with positive validation in the comparison to the yields of the MC simulation had proved that a linear model was sufficiently accurate. In the present study the results were directly compared for the same number of the iterations (3), in order to check the impact of the more complicated second-order model on the probabilistic estimations.

The first point of discussion regards the computational efficiency of the probabilistic numerical methods. The most conventional approach to reliability assessment is undoubtedly the MC sampling method, however, it is well known to be highly computationally expensive. It was run with the same input data regarding the random variables distributions (Table 1) and the deterministic constants and it took many hours for the completion of one million iterations. From the point of view of the probabilistic estimations, it proved to be able to estimate probabilities greater than $10^{-6}$, with a rough approximation at the lowest levels between $10^{-6}$ and $10^{-5}$. According to several references dealing with MC simulation, such as [3], it was completely unsuitable to estimate probabilities lower than $10^{-6}$, due to the fixed number of samplings. Of course, it would be possible to shift this threshold 
downwards, by incrementing the number of required samplings but it would unacceptably increase the computational time: e.g., going down to a $10^{-7}$ threshold would require at least 10 million iterations, with an exponential increment of the required time.

The probabilities of failure and the safety indexes at different last stages were point by point determined by the numerical first- and second-order methods. For this purpose, a number of 20 points, corresponding to lives in the range from 1,000 to 100,000 cycles, was considered. The iterative algorithm described in the previous paragraph was run for each of the 20 points, utilizing both the linear and the non linear models. The two approaches proved to be very efficient: as mentioned, the computation converged after only three iterations, corresponding to a very short computational time: just 5 seconds for the linear model and 7 seconds for the non linear one. Moreover, both the models were able to easily compute even very low probabilities of failure at the machine life stages, as the CRRs must be designed with very high safety requirements and high reliability. The computational full time for the determination of the two curves is consequently between 2 and 3 minutes, which is many orders of magnitude lower than the time required by a MC simulation.

Figs. 9-10 show the obtained results in terms of the safety index and of the probability of failure: the results are plotted considering a life range from 5,000 cycles, which is the most interesting to investigate, as it refers to the second part of the machine life. Moreover, different symbols are used to indicate the yields of the first- and of the second-order models. The results of the MC simulation in terms of probability of failure vs. life cycles are added in Fig. 10, where the CDFs are compared. What can be observed is that the results for the linear and the non linear model are almost coincident, with differences in the order of few percentage points that can be therefore neglected. It leads to the conclusion that the impact of the increasing complexity of the second-order expansion is negligible in this case. The first order model is therefore to be preferred, as it is much easier to develop and has the same accuracy: this result is also a confirmation of the conclusions of [2].

Dealing with safety index results, it can be remarked that $\beta$ assumes values higher than 6.5 in the machine life range. This amount can be compared to that reported in [5] with reference to the probabilistic design of nuclear pipes. In this Reference, based on the literature in the field, the target reliability index (minimum desired value) was set to 3 . In other contributions, in particular in [35-36], safety index reference ranges are reported for the fatigue design of different structures. For instance, in the case of buildings or bridges, the suggested interval is between 1.5 and 3.8, while in ship de- 
sign the target safety index is usually between 2 and 3.5. This comparative analysis leads to the conclusion that the obtained values seem to be about $50 \%$ greater than the reference values in literature for structure under fatigue. A possible reason for the studied CRR having so high safety issues is related to the serious consequences for human health and for the environment in the case of CRR explosion. However, this outcome was a motivation to deepen the analysis with the computation of the probability of failure and with its comparison to other literature data.

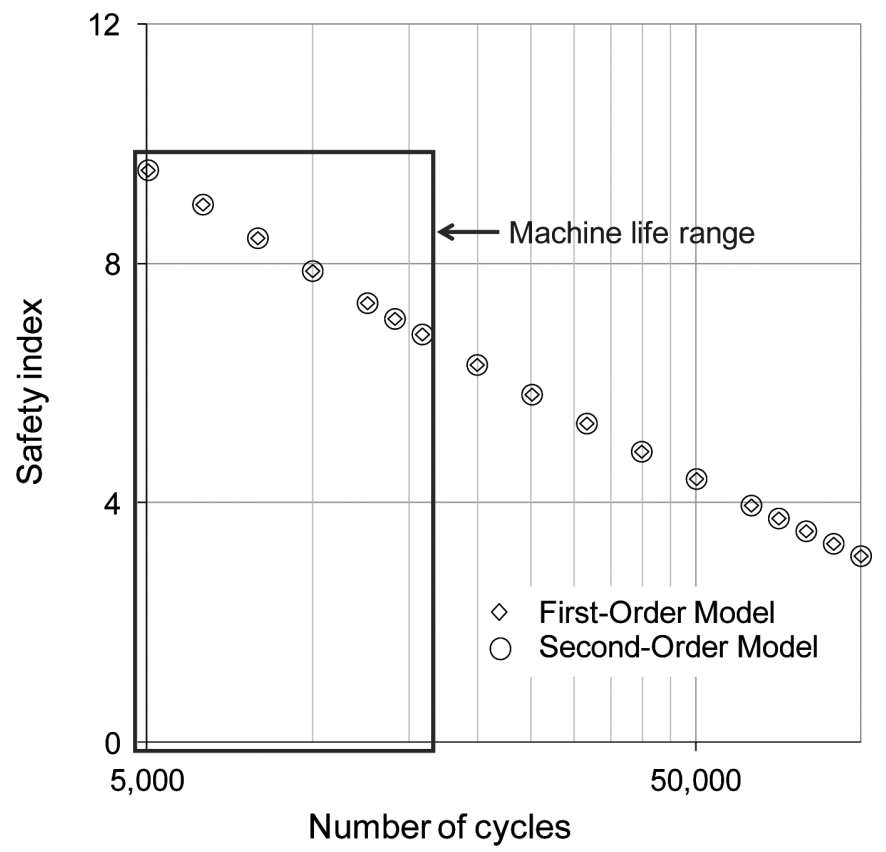

Fig. 9. Safety index-life cycles curve determined by First- and Second-Order Models

From the quantitative point of view, the estimated probability of failure is about $1 \cdot 10^{-19}$ after 6,000 loops, $2 \cdot 10^{-17}$ at 7,900 loops and increases up to $2 \cdot 10^{-15}$ after 10,000 loops and to $4 \cdot 10^{-12}$ at 15,000 loops, i.e. at the end of the turbogenerator fifty-year life. This values are in the typical order of magnitude of the probability of failure of highly loaded components under LCF, whose breakage may have very serious consequences. For example, the study in [6] deals with the probabilistic analysis of an aeronautical engine turbine disc structure, which leads to the estimation of probabilities of failure in the range $10^{-16}-10^{-8}$ at the most loaded sections. 


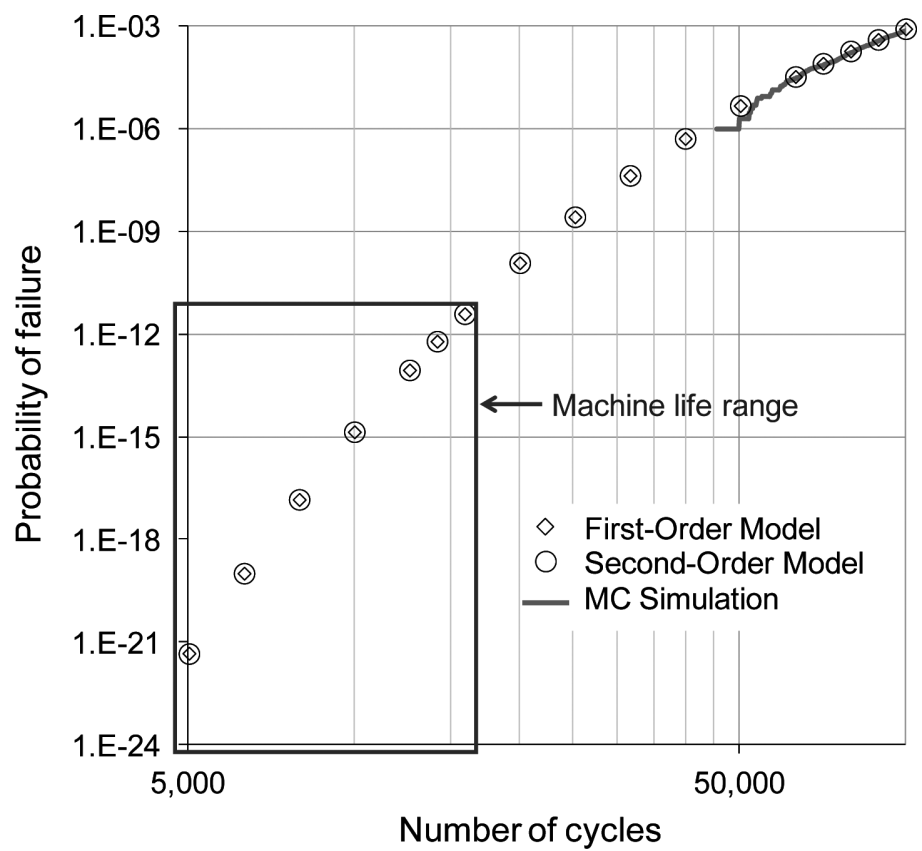

Fig. 10. Probability of failure-life cycles curve determined by First- and Second-Order Models and by MC Simulation

\section{Conclusions}

The present paper dealt with the determination of the probability of failure of a turbogenerator CRR: the reliability assessment was conducted with reference to the most critical failure mode (CRR swelling in tension under centrifugal force). The problem was tackled from the numerical point of view, with analytical and statistical processing of experimental data. The main points can be summarized as follows.

- The analysis of literature showed that, despite the so many safety issues related to turbogenerator design, there are few papers dealing with this subject. Moreover, from the methodological point of view, the main steps for the application of numerical methods, such as the AMV, are usually not described.

- The CRR material (18Mn18Cr) was experimentally characterized for the determination of its static, cyclic and LCF curves, its Masing behaviour was also experimentally proved. A simple statistical methodology was introduced to estimate the stochastic distributions of the material parameters, according to the Ramberg-Osgood and Manson-Coffin models, and on the basis of experimental data.

- Eight input random variables were considered. The scatter of the static and cyclic curves, as well as that of the LCF, were taken into account, 
thus meeting the recommendations of literature. Moreover, this approach made it possible to consider the random properties of the local stressstrain states in the elastic-plastic field.

- Numerical algorithms were developed for the determination of the probability of failure, according to the AMV approach. As a first step, a Taylor polynomial expansion was determined to relate the input variables to the result in terms of the CRR life. This functional relationship was determined according to two models: a linear and a not linear one.

- The numerical models (both linear and second order) proved to be very efficient and accurate: the CDF of the probability of failure could be determined in very few minutes, with result convergence after just three iterations. On the other hand, the tool of MC simulation proved to be much more inefficient in the estimation of low probabilities of failure. In the direct comparison between the first and the second order models, no difference could be observed between results. It shows that the simpler first order model is sufficient to manage the uncertainties in this study.

- From the quantitative point of view, the safety index of the studied device is higher than 6.5, which indicates that the safety requirements are fully accomplished. The probability of failure at machine full life is in the order of $10^{-12}$. This value is consistent with reference data for components in the mechanical and aeronautical field and is justified by the very serious outcomes of CRR explosion.

- The applied method (both first- and second order models) may be extended to many other engineering applications. For instance, in the turbogenerator field, it can be used for the probabilistic assessment of copper connectors. Other possible applications regard turbine disks [6] or blades [37], or piping systems [5, 34].

\section{Manuscript received by Editorial Board, March 13, 2013;} final version, September 02, 2013.

\section{REFERENCES}

[1] Olmi G.: Dalla sperimentazione a fatica oligociclica alla valutazione della probabilità di guasto di un rotore di un turboalternatore (in Italian), Proc. $40^{\text {th }}$ AIAS National Conference, Palermo (Italy), 2011, pp. 1-13.

[2] Olmi G.: An Efficient Method for the Determination of the Probability of Failure on the Basis of LCF Data: Application to Turbogenerator Design. Structural Durability \& Health Monitoring, 2012, Vol. 8, No. 1, pp. 61-89.

[3] Huang B., Du X.: Probabilistic uncertainty analysis by mean-value first order Saddlepoint Approximation. Reliability Engineering and System Safety, 2008, Vol. 93, No. 2, pp. 325336.

[4] Wu Y.T., Millwater H.R., Cruse T.A.: Advanced probabilistic structural analysis method for implicit performance functions. AIAA Journal, 1990, Vol. 28, No. 9, pp. 1663-1669. 
[5] Avrithi K., Ayyub B.M.: A Reliability-Based Approach for Low-Cycle Fatigue Design of Class 2 and 3 Nuclear Piping. Journal of Pressure Vessel Technology, 2010, Vol. 132, No. 5, pp. 051202-1-6.

[6] Liu C.L., Lu Z.Z., Xu Y.L., Yue Z.F.: Reliability analysis for low cycle fatigue life of the aeronautical engine turbine disc structure under random environment. Materials Science and Engineering A, 2005, Vol. 395, No. 1-2, pp. 218-225.

[7] Olmi G.: Utilizzo di metodi non lineari nella valutazione affidabilistica di dispositivi meccanici con più variabili aleatorie (in Italian), Proc. $40^{\text {th }}$ AIAS National Conference, Palermo (Italy), 2011, pp. 1-15.

[8] Wu Y.T., Wirsching P.H.: Advanced Reliability Method for Fatigue Analysis. Journal of Engineering Mechanics, 1984, Vol. 110, No. 4, pp. 536-553.

[9] Olmi G., Freddi A.: LCF on turbogenerator rotors and coil retaining rings: material characterization and sensitivity analyses. Proc. ICEM14 $-14^{\text {th }}$ International Conference on Experimental Mechanics, Poitiers (France), 2010, Vol. 1, pp. 1-9.

[10] Olmi G.: Low Cycle Fatigue experiments on turbogenerator steels and a new method for defining confidence bands. Journal of Testing and Evaluation (JTE), 2012, Vol. 40, No. 4, pp. 539-552.

[11] Olmi G.: A Novel Method for Strain Controlled Tests. Experimental Mechanics, 2012, Vol. 52, No. 4, pp. 379-393.

[12] Olmi G.: A new loading-constraining device for mechanical testing with misalignment autocompensation. Experimental Techniques, 2011, Vol. 35, No. 6, pp. 61-70.

[13] Orita K., Ikeda Y., Iwadate T., Ishizaka J.: Development and production of $18 \mathrm{Mn}-18 \mathrm{Cr}$ nonmagnetic retaining ring with high yield strength. ISIJ J, 1990, Vol. 30, No. 8, pp. 587-593.

[14] Balitskii A., Krohmalny O., Ripey I.: Hydrogen cooling of turbogenerators and the problem of rotor retaining ring materials degradation. International Journal of Hydrogen Energy, 2000, Vol. 25, No. 2, pp. 167-171.

[15] International Organization for Standardization ISO 12106:2003(E): Metallic materials-Fatigue testing-Axial-strain-controlled method, 2003, Geneva (Switzerland).

[16] Doyle J.F.: Modern Experimental Stress Analysis. West Sussex (England), John Wiley and Sons Ltd., 2004.

[17] Wirsching P.H., Torng T.Y., Martin W.S.: Advanced fatigue reliability analysis. International Journal of Fatigue, 1991, Vol. 13, No. 5, pp. 389-394.

[18] Grujicic M., Arakere G., Bell W.C., Marvi H., Yalavarthy H.V., Pandurangan B., Haque I., Fadel G.M.: Reliability-Based Design Optimization for Durability of Ground Vehicle Suspension System Components. Journal of Materials Engineering and Performance, 2010, Vol. 19, No. 3, pp. 301-313.

[19] Choi K.K., Youn B.D.: On Probabilistic Approaches for Reliability-Based Design Optimization. Proc. 9th AIAA/NASA/USA/ISSMO Symposium on Multidisciplinary Analysis and Optimization, Atlanta, GA (USA), 2002.

[20] Zhu S.-P., Huang H.-Z., Ontiveros V., He L.-P., Modarres M.: Probabilistic Low Cycle Fatigue Life Prediction Using an Energy-Based Damage Parameter and Accounting for Model Uncertainty. International Journal of Damage Mechanics, 2012, Vol. 21, No. 8, pp. 1128-1153.

[21] Zhang Z.P., Qiao Y.J., Sun Q., Li C.W., Li J.: Theoretical Estimation to the Cyclic Strength Coefficient and the Cyclic Strain-Hardening Exponent for Metallic Materials: Preliminary Study. Journal of Materials Engineering and Performance, 2009, Vol. 18, No. 3, pp. 245-254.

[22] McDaniels R.L., Chen L., Steward R., Liaw P.K., Buchanan R.A., White S., Liaw K., Klarstrom D.L.: The strain-controlled fatigue behavior and modeling of Haynes ${ }^{\circledR}$ HASTELLOY ${ }^{\circledR}$ C-2000 ${ }^{\circledR}$ superalloy. Materials Science and Engineering A, 2011, Vol. 528, No. 12, pp. 3952-3960.

[23] Ellyin F.: Fatigue Damage, Crack Growth, and Life Prediction. London (England), Chapman and Hall, 1997. 
[24] Ellyin F.: Cyclic Strain Energy Density as a Criterion for Multiaxial Fatigue Failure, Biaxial and Multiaxial Fatigue. London (England), Mechanical Engineering Publications Limited, 1989.

[25] Suresh S.: Fatigue of Materials. Cambridge (United Kingdom), Cambridge University Press, 1991.

[26] Dowling N.E.: Mechanical Behavior of Materials (3rd edition). Prentice-Hall, 2006.

[27] Ellyin F.: Effect of Tensile-Mean-Strain on Plastic Strain Energy and Cyclic Response. Journal of Engineering Materials and Technology, 1985, Vol. 107, No. 2, pp. 119-125.

[28] Dominique F.: Structural Components: Mechanical Tests and Behavioral Laws. Wiley-ISTE, 2008.

[29] Kang G., Gao Q., Yang X.: Uniaxial cyclic ratcheting and plastic flow properties of SS304 stainless steel at room and elevated temperatures. Mechanics of Materials, 2002, Vol. 34, No. 3, pp. 145-159.

[30] Dingli J.P., Abdul-Latif A., Saanouni K.: Predictions of the complex cyclic behavior of polycrystals using a self-consistent modeling. International Journal of Plasticity, 2000, Vol. 16, No. 3-4, pp. 411-437.

[31] Alvarez-Armas I., Degallaix-Moreuil S.: Duplex Stainless Steels. Wiley-ISTE, 2009.

[32] Khutia N., Dey P.P., Kumar Paul S., Tarafder S.: Development of non Masing characteristic model for LCF and ratcheting fatigue simulation of SA333 C-Mn steel. Mechanics of Materials, 2013, Vol. 65, pp. 88-102.

[33] Jiang J., Fan Z.: Fatigue behavior of pressure vessel steels at elevated temperature. Journal of China Pressure Vessel Technology, 2003, Vol. 1, pp. 109-114.

[34] Zhao Y.X.: A methodology for strain-based fatigue reliability analysis. Reliability Engineering and System Safety, 2000, Vol. 70, No. 2, pp. 205-213.

[35] ENV 1991-1 (Eurocode 1): Basis of design and actions on structures - Part I: Basis of design. Brussels, CEN (Comite Europien de la Normalisation), 1994.

[36] Mansour A.E., Wirsching P.H., White G.J., Ayyub B.M.: Probability-Based Ship Design: Implementation of Design Guidelines. Washington, DC, NTIS, Report No. SSC 392, 1992.

[37] Vyas N.S., Sidharth Rao J.S.: Dynamic stress analysis and a fracture mechanics approach to life prediction of turbine blades. Mechanism and Machine Theory, 1997, Vol. 32, No. 4, pp. 511-527.

\section{Ocena niezawodności pierścieni ustalających cewek turbogeneratora w oparciu o dane zmęczeniowe przy małej liczbie cykli}

$$
\text { Streszczenie }
$$

Pierścienie ustalające cewek turbogeneratora są pasowane skurczowo na wirniku wokół cerek by zabezpieczyć je przed działaniem sił odśrodkowych. W typowych warunkach, są one poddane narażeniom zmęczeniowym o malej liczbie cykli, przy czym każdy cykl rozpoczyna się od startu maszyny, a kończy przy jej zatrzymaniu. Przedmiotem artykułu jest wyznaczenie prawdopodobieństwa awarii pierścienia ustalającego cewki. Rozważanym uszkodzeniem jest pęknięcie pierścienia spowodowane puchnięciem materiału pod wpływem naprężeń wywołanych silą odśrodkową. Ocena niezawodności przeprowadzona w tej pracy wykorzystuje dane wejściowe wpływające na obciążenie zmęczeniowe przy małej liczbie cykli i ich rozkłady stochastyczne. Dla rozwiązania problemu wyznaczono eksperymentalnie charakterystyki statyczne i krzywe cykli zmęczeniowych stosowanego materiału i zastosowano model statystyczny dla wyliczenia odpowiednich parametrów i ich odchyleń standardowych. Na podstawie wyznaczonych rozkładów zmiennych estymuje się prawdopodobieństwo uszkodzenia w formie dystrybuanty rozkładu. Wykorzystany 
algorytm obliczeniowy o dużej skuteczności wykorzystuje metodę "zaawansowanej średniej" (Advanced Mean Value). Uzyskane wyniki dają informację o odpowiedzi materiału i stanie lokalnych naprężeń i odkształceń w najbardziej obciążonych obszarach pierścienia ustalającego cewki. Prawdopodobieństwo uszkodzenia, wyznaczone na koniec okresu eksploatacji maszyny, wynosi $10^{-12}$ $\mathrm{i}$ jest porównywalne $\mathrm{z}$ wartościami referencyjnymi dla innych konstrukcji mechanicznych i lotniczych narażonych na zmęczenie. 